\title{
Analytical Study of the Process of Dissolution of Silicon from a Modifier Particle
}

\author{
Denis Boldyrev ${ }^{1}$, Maksim Kharchenko ${ }^{2, *}$, Ruslan Amirov², and Anton Bokov ${ }^{2}$ \\ ${ }^{1}$ Department of Nanotechnology, Materials Science and Mechanics, Togliatti State University, \\ 445020 Togliatti, Russia; \\ ${ }^{2}$ Department of Metal forming machines and technologies, Nosov Magnitogorsk State Technical \\ University, 455000 Magnitogorsk, Russia
}

\begin{abstract}
The process of dissolution of a particle of a ferrosilicon modifier in a cast iron melt is considered, taking into account such a physical analogy as the distribution of heat from a point source in space (an infinite body).
\end{abstract}

\section{Introduction}

An increase in the mechanical properties of cast iron is achieved mainly by changing the shape, size and distribution of graphite inclusions. Modification of melts has a significant impact on the processes of graphitization of cast iron [1-4]. Modification of gray cast iron with graphitizing additives reduces its tendency to chill, eliminates interdendritic graphite, and reduces the size of graphite petals [5-9]. The applied modifiers can be conditionally divided into graphitizing, stabilizing the cementite phase and spheroidizing. Graphitizing modifiers stimulate the process of graphitization during eutectic transformation through the formation of additional centers of graphite crystallization. With an increase in their number, the supercooling of the melt before the solidification of the eutectic decreases, which helps to prevent chilling of the surface layer of castings and the structure of the supercooled graphite eutectic. Stabilizing modifiers have practically no effect on the graphitization process during the solidification of cast iron. They are used to preserve the cementite phase after eutectic transformation, as a result of which the pearlite content in the structure of castings increases, providing an increase in strength characteristics. Spheroidizing modifiers provide a change in the shape of graphite towards its approximation to the globular one. Typically, spheroidizing modifiers are used to produce vermicular and nodular cast irons.

In most cases, in the practice of foundry use complex modifiers, which include magnesium, silicon, lithium, aluminum, calcium, manganese, beryllium, cerium, yttrium and other rare earth metals (REM). Nickel, copper, silicon or calcium are usually used as the base of the ligature. A whole range of requirements are imposed on the composition of ligatures, among which the following can be distinguished:

- high modifying ability at relatively low doses of the modifier;

\footnotetext{
*Corresponding author: kharchenko.mv@bk.ru
} 
- regulated density of the master alloy, ensuring its high degree of assimilation with relatively simple methods of introduction into the melt;

- minimal smoke emission and prevention of pyroeffects during the introduction of ligatures;

- regulated "survivability" of the modifier, ensuring the achievement of the modifying effect directly in the finished casting to the maximum extent.

Graphitizing modifiers conditionally, depending on the silicon content, can be divided into two classes: siliceous $(\mathrm{Si} \geq 15 \%)$ and non-siliceous ( $\mathrm{Si}$ is absent or is contained in an amount of less than $15 \%$ ). There are many works on the kinetics of dissolution of modifiers based on the mass balance of a substance [10-17].

\section{Methods}

To conduct an analytical study of the concentration field of silicon distribution from a ferrosilicon modifier particle dissolving in a cast iron melt, we use a physical analogy with such a process as the distribution in space (in an infinite body) of heat from a point source.

The solution to this problem should be a function of the following form $\mathrm{C}_{\mathrm{Si}}(\mathrm{x}, \mathrm{y}, \mathrm{z}, \mathrm{t})=\mathrm{f}$ $(\mathrm{x}, \mathrm{y}, \mathrm{z}, \mathrm{t})$, which characterizes the accumulation of silicon $\mathrm{C}_{\mathrm{Si}}$ at any time. Finding such a function is a solution to the differential equation taking into account the boundary and initial conditions, as well as the geometric shape of the body.

When considering the calculation method for solving this problem, an analytical method can be applied, which consists in determining the equation of the process corresponding to the differential equation of the distribution of the concentration of a substance and the boundary conditions. The simplest source method of all analytical methods will be used to solve the original problem. The physical essence of this method assumes that any process of heat propagation or concentration of a substance in a medium can be represented by a set of processes of temperature or concentration equalization from many elementary heat sources distributed in space and time. Solving problems using this method consists in the correct selection of sources and their distribution.

Assume such an ideal theoretical scheme as an infinite body (Fig. 1) having a length along the axes $\mathrm{OX}, \mathrm{OY}, \mathrm{OZ}$, in which the boundaries do not influence on the distribution of concentration of a substance to conduct calculations. In other words, the body has unlimited extension in all 3 directions.

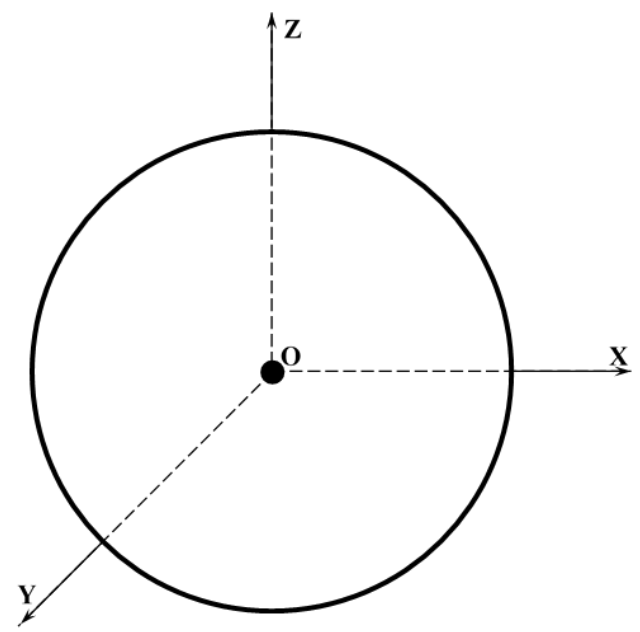

Fig. 1. Design scheme - point source in an infinite body 
The heat source under study is classified as a point heat source with an infinitely small volume, instantaneously acting, representing a point in the limit. That is, the source tends to zero duration of action and is stationary, not moving in the body (or body). At the initial moment of time $t=0$ in it, in an infinitely small volume element $d x, d y, d z$, located at the initial zero temperature $\mathrm{T} 0=0$, there is (concentrated) a certain amount of heat $\mathrm{Q}[\mathrm{J}]$. The thermophysical properties of a body are determined by the thermal conductivity coefficient $\lambda\left[\mathrm{J} / \mathrm{cm} \mathrm{sec}{ }^{\circ} \mathrm{C}\right]$, the volumetric heat capacity $\mathrm{c} \gamma\left[\mathrm{J} / \mathrm{cm}^{3}{ }^{\circ} \mathrm{C}\right]$ and the thermal diffusivity coefficient a $[\mathrm{cм} \cdot \Gamma /$ сек], which remain constant throughout the body during the entire process and independent of temperature. For the chosen model, the heat propagation process is expressed by the equation:

$$
T(R, t)=\frac{Q}{c \gamma(4 \pi a t)^{3 / 2}} e^{\frac{R^{2}}{4 a t}},
$$

where $\mathrm{R}^{2}=\mathrm{x}^{2}+\mathrm{y}^{2}+\mathrm{z}^{2}$ is the square of the distance from the heat source $\mathrm{O}$ to the point of the body with coordinates $\mathrm{x}, \mathrm{y}, \mathrm{z}$. This process is symmetric about point $\mathrm{O}$, that is, the temperature of any point of the body is determined only by its spherical radius vector $\mathrm{R}$. Isothermal surfaces are spheres $\mathrm{R}=$ const centered at a point source $\mathrm{O}$.

At $t=0$, according to the equation, the point $\mathrm{O}, \mathrm{T}(0,0,0) \rightarrow \infty$ is heated to an infinitely high temperature, since at the initial moment, the finite amount of heat $\mathrm{Q}$ is concentrated at a point, that is, in an infinitely small volume element. In this case, the entire rest of the volume of the body outside the point source has a zero initial temperature $T(R, 0)=0$, and at points of the body $\mathrm{R} \rightarrow \infty$ far from the source, the temperature remains invariably equal to zero, $\mathrm{T}(\infty, \mathrm{t}) \rightarrow 0$. The temperature from the source decreases in the medium along isothermal spherical surfaces and is expressed by a factor:

and the factor:

$$
e^{\frac{R^{2}}{4 a t}}
$$

$$
\frac{Q}{c \gamma(4 \pi a t)^{3 / 2}},
$$

characterizes the temporal decrease in the temperature of the point $\mathrm{R}=0$ with the highest temperature.

Taking into account that the modifier particles are introduced en masse into the cast iron melt during the modification operation, the model under consideration must necessarily take into account the principle of superposition (superposition) from a variety of point instantly acting stationary sources. That is, heat from each source is distributed in the environment regardless of the action of other identical sources. Isothermal surfaces of individual sources are superimposed on each other without interacting with each other. This principle is that the temperature in the process of heat propagation under the joint action of the mass of sources is considered as the sum of temperatures from the action of each individual source.

Transferring the considered analogy of a point instantaneously acting stationary heat source to a ferrosilicon modifier particle dissolving in the cast iron melt, the thermal diffusivity in equation (1) changes to the diffusion coefficient $D$. Thus, $C_{S i}(R, t)$ is the concentration of silicon in the cast iron melt at a distance $\mathrm{R}$ from the modifier particle, reached after time $t$. The propagation of silicon concentration is expressed by the following equation (2):

$$
T(R, t)=\frac{Q}{c \gamma(4 \pi a t)^{3 / 2}} e^{\frac{R^{2}}{4 a t}}
$$




\section{Conclusion}

Thus, the obtained equation for the propagation of silicon concentration can be used to analyze the process of dissolution of silicon modifiers in a cast iron melt.

It is also worth noting that it is necessary to check the reliability of the obtained equation using experimental data.

This work is carried out within a framework of the government order (No. FZRU-2020-0011) of the Ministry of Science and Higher Education of the Russian Federation.

\section{References}

1. V.I. Vozdvizhenskij, V.A. Grachev and V.V. Spasskij, Foundry alloys and their melting technology in mechanical engineering (Mashinostroenie, Moscow, 1984)

2. R.D. Joseph, ASM Specialty Handbook: Cast Irons (ASM International, 1996)

3. A.P. Truhov and A.I. Malyarov, Foundry alloys and smelting (Akademiya, Moscow, 2004)

4. Sv.S. Kvon, V.Yu. Kulikov, Ye.P. Shcherbakova, S. K. Arinova, Metalurgija, 58(3-4), 315-318 (2019)

5. V.A. CHajkin, D.A. Boldyrev and N.V. CHajkina, Vestnik of NMSTU, 4 53-58 (2007)

6. K.N. Vdovin, M.V. Shubina, I.G. Shubin, Solid State Phenomena, 284, 332-337 (2018)

7. D.A. Boldyrev, A.N. Shapovalov, S.P. Nefedev, R.R. Dema, V.N. Kononov, M.V. Kharchenko, E.V. Suvorova and I.Yu. Markova, J. Chem. Technol. Metall. 54, 348361 (2019)

8. D.A. Boldyrev, S.I. Platov, N.V. Urtsev, D.V. Terentyev and O.R. Latypov, IOP Conf. Series: Materials Science and Engineering, 1008, 012047(2020)

9. V.N. Urtsev, A.V. Shmakov, N.V. Urtsev, E.D. Mokshin, D.M. Khabibulin, V.N. Degtyarev, P.A. Stekanov, Y.N. Gornostyrev, M.L. Lobanov, S.I. Platov, K.B. Maslennikov, Steel in Translation 50(7), 496-500 (2020)

10. D.A. Boldyrev, R.N. Amirov and S.P. Nefed'ev, IOP Conf. Series: Materials Science and Engineering, 966 012023(2020)

11. V. Ivanov, V. Pirozhkova, V. Lunev, East.-European J. Ent. Tech. 4/12 88, 26-30 (2017)

12. D.M. Stefanescu, Mater. Sci. Eng. A 413-414, 322-333 (2005)

13. J. Mallia, M. Grech and R.E. Smallman, Materials Science and Technology, 14:5, 452-460 (1998)

14. P.S. Harlashin, V.YA. Bakst, A.V. Bendich, Vestnik Priazovskogo gosudarstvennogo tekhnicheskogo universiteta. Seriya: Tekhnicheskie nauki, 25, 13-20 (2012)

15. S.N. Lekakh, B. Hrebec, Solidification Kinetics of Graphite Nodules in Cast Iron and Shrinkage Porosity, Int. J. Met. 10, 389-400 (2016)

16. G. Alonso, P. Larrañaga, D. M. Stefanescu, E. De la Fuente, A. Natxiondo, R. Suarez, Int. J. Met. 11, 14-26 (2017)

17. YU.V. Erohin, A.V. Zaharov, L.V. Leonova, Vestnik of NMSTU, 18, 13-21 (2020) 